\title{
RICCATI TECHNIQUES AND VARIATIONAL PRINCIPLES IN OSCILLATION THEORY FOR LINEAR SYSTEMS
}

\author{
G. J. BUTLER, L. H. ERBE AND A. B. MINGARELLI
}

\begin{abstract}
We consider the seond order differential system (1) $Y^{\prime \prime}+Q(t) Y=$ 0 , where $Q, Y$ are $n \times n$ matrices with $Q=Q(t)$ a continuous symmetric matrixvalued function, $t \in[a,+\infty)$. We obtain a number of sufficient conditions in order that all prepared solutions $Y(t)$ of $(1)$ are oscillatory. Two approaches are considered, one based on Riccati techniques and the other on variational techniques, and involve assumptions on the behavior of the eigenvalues of $Q(t)$ (or of its integral). These results extend some well-known averaging techniques for scalar equations to system (1).
\end{abstract}

1. Introduction. Consider the second order differential system

$$
Y^{\prime \prime}+Q(t) Y=0, \quad t \in[a,+\infty),
$$

where $Y(t), Q(t)$ are $n \times n$ real continuous matrix functions with $Q(t)$ symmetric. The vector system associated with (1.1) is

$$
y^{\prime \prime}+Q(t) y=0
$$

where $y=\operatorname{col}\left(y_{1}, \ldots, y_{n}\right)$ is an $n$-vector. Equation (1.2) is said to be disconjugate on an interval $J \subset[a,+\infty)$ if every nontrivial solution of (1.2) vanishes at most once in $J$ and (1.2) is said to be oscillatory if for each $t_{0}>a$ there exists $t_{1}>t_{0}$ such that (1.2) is not disconjugate on $\left[t_{0}, t_{1}\right]$. A solution $Y(t)$ of the matrix equation (1.1) is said to be nontrivial if $\operatorname{det} Y(t) \neq 0$ for at least one $t \in[a,+\infty)$ and a nontrivial solution $Y(t)$ is said to be prepared or self-conjugate in case

$$
Y^{*}(t) Y^{\prime}(t)-Y^{* \prime}(t) Y(t) \equiv 0, \quad t \in[a,+\infty),
$$

(where for any matrix $A$, the transpose of $A$ is denoted by $A^{*}$ ). Note that for any solution $Y(t)$ of (1.1) the expression on the left of (1.3) is constant. Equation (1.1) is said to be oscillatory on $[a,+\infty)$ in case the determinant of every nontrivial prepared solution vanishes on $[b,+\infty)$ for each $b>a$. This is equivalent to oscillation of equation (1.2) since any solution of (1.2) is of the form $y(t) \equiv Y(t) \alpha$ for some constant vector $\alpha$ and some nontrivial prepared solution $Y(t)$ of $(1.1)$.

The oscillation theory for the corresponding scalar equation

$$
y^{\prime \prime}+q(t) y=0, \quad t \in[a,+\infty),
$$

Received by the editors January 23, 1985 and, in revised form, January 31, 1986 and September 16, 1986.

1980 Mathematics Subject Classification. Primary 34C10.

Key words and phrases. Oscillation, Riccati equation, variational techniques.

Research supported by grants from NSERC-Canada.

Dedicated to the memory of Geoffrey J. Butler, who passed away on July 13, 1986, during the final preparation of this paper. 
where $q(t)$ is a real-valued continuous function has a very extensive literature and there are a number of survey papers which have been written, $[\mathbf{2 7}, \mathbf{3 0}, \mathbf{2 3}]$-see also the book of Swanson [24]. The corresponding theory for (1.1) and (1.2) is less developed although there have been quite a number of papers devoted to attempts at paralleling the scalar theory. Many recent results $[\mathbf{1 3}, \mathbf{7}, \mathbf{8}, \mathbf{2 6}, \mathbf{1 1}, \mathbf{2}, \mathbf{2 5}$, have concentrated on showing that (1.1) is oscillatory if a corresponding scalar equation obtained by applying a positive linear functional is oscillatory. That is, if we denote by $S$ the linear space of all $n \times n$ real symmetric matrices, then a linear functional $\varphi: S \rightarrow(-\infty,+\infty)$ is said to be positive if $\varphi(A) \geq 0$ for $A \in S$ and $A \geq 0$ (i.e., $A$ symmetric and positive semidefinite). The basic result obtained (cf. $[\mathbf{1 1}, \mathbf{1 3}])$ is that $(1.1)$ (or $(1.2))$ is oscillatory on $[a,+\infty)$ in case there exists a positive linear functional $\varphi$ with $\varphi(I)=1$ ( $I=$ identity matrix) such that the scalar equation

$$
u^{\prime \prime}+\varphi(Q(t)) u=0
$$

is oscillatory.

Several other recent oscillation criteria for (1.1) have been given which involve the eigenvalues of $Q(t)$ (or of its integral). For any real symmetric matix $A$, we will assume its eigenvalues $\lambda_{k}(A), 1 \leq k \leq n$, are ordered so that

$$
\lambda_{1}(A) \geq \lambda_{2}(A) \geq \cdots \geq \lambda_{n}(A),
$$

and as usual,

$$
\operatorname{tr} A=\sum_{i=1}^{n} \lambda_{i}(A) .
$$

For the scalar equation (1.4) the well-known Fite-Wintner Theorem $[\mathbf{9}, \mathbf{3 0}]$ states that (1.4) is oscillatory if

$$
\int_{a}^{\infty} q(t) d t=+\infty
$$

and hence it is clear that (1.1) is oscillatory if

$$
\int_{a}^{\infty} \operatorname{tr} Q(t) d t=+\infty .
$$

Mingarelli in $[18,19]$ showed that the positive linear functional $\operatorname{tr} A$ can be replaced by the convex function $\lambda_{1}(A)$, provided a certain growth condition on $\int_{a}^{t} \operatorname{tr} Q(s) d s$ is assumed. This answered, in part, a conjecure [13] which was also considered in the thesis of Akiyama [1]. Additional results which extend and improve some of these criteria were also obtained in $[\mathbf{4}, \mathbf{5}, \mathbf{1 4}, \mathbf{1 7}]$.

Finally, it has recently been shown by Byers, Harris and Kwong [6] that

$$
\lim _{t \rightarrow \infty} \lambda_{1}\left(\int_{a}^{t} Q(s) d s\right)=+\infty
$$

without any additional conditions, is an oscillation criterion for (1.1). This gives, therefore, the desired systems analogue of the Fite-Wintner condition.

It is the aim of this paper to apply two of the fundamental approaches used in studying the scalar equation (1.4)--namely the Riccati integral equation approach and the variational approach - to obtain a number of oscillation tests for (1.1). We 
refer to the recent work of Kwong and Zettl [15] and the references therein where these two approaches are considered for the scalar equation.

In $\S \S 2$ and 3 we present several oscillation criteria which may be regarded as generalizations to systems of various well-known scalar tests, other then the FiteWintner criterion. In $\S 2$ the proofs are based on the Riccati equation technique, whereas in $\S 3$ we discuss oscillation criteria which are proved using variational techniques. In $\S 4$ we present several examples showing that certain subsidiary hypotheses for these theorems are necessary. The proofs of the results are given in $\S \S 5$ and 6.

In a strictly formal sense, any oscillation or nonoscillation theorem for (1.1) should be accessible by either the Riccati or variational method. In practice, by considering these two different approaches, one seems to gain greater insight into the way in which conditions imposed on the coefficient matrix $Q$ or its integral contribute to the oscillatory nature of the equation. This is one of the purposes of this present paper. Our other purpose is to illustrate the extent to which scalar oscillation criteria can be generalized to systems, utilizing the behavior of the largest eigenvalue and thereby achieving sharper results than can be obtained by the use only of positive linear functionals such as the trace.

We recall here some notation and definitions which will be useful in the sequel. For any subset $E$ of the real line $R, \mu(E)$ denotes the Lebesgue measure of $E$. If $f(t)$ denotes a continuous real-valued function and if $l ; m$ satisfy $-\infty \leq l, m \leq+\infty$, then we say limapprox $\inf _{t \rightarrow \infty} f(t)=l$ in case $\mu\left\{t: f(t) \leq l_{1}\right\}<+\infty$ for all $l_{1}<l$ and $\mu\left\{t: f(t) \leq l_{2}\right\}=+\infty$ for all $l_{2}>l$. Similarly, lim approx $\sup _{t \rightarrow \infty} f(t)=m$ in case $\mu\left\{t: f(t) \geq m_{1}\right\}=+\infty$ for all $m_{1}<m$ and $\mu\left\{t: f(t) \geq m_{2}\right\}<+\infty$ for all $m_{2}>m$. Finally, $\lim _{\text {approx }} \rightarrow \infty f(t)=\lambda$ in case

$$
\lim _{t \rightarrow \infty} \operatorname{approx} \sup f(t)=\lim _{t \rightarrow \infty} \operatorname{approx} \inf f(t)=\lambda .
$$

2. Riccati techniques. We recall [12] that the scalar equation (1.4) is nonoscillatory on $[a,+\infty)$ if and only if the Riccati integral equation

$$
r(t)=r\left(t_{0}\right)+\int_{t_{0}}^{t} q(s) d s+\int_{t_{0}}^{t} r^{2}(s) d s
$$

has a continuous solution on $\left[t_{0}, \infty\right)$ for some $t_{0} \geq a$; likewise the matrix equation (1.1) is nonoscillatory on $[a,+\infty)$ if and only if the corresponding matix equation

$$
Z(t)=Z\left(t_{0}\right)+\int_{t_{0}}^{t} Q(s) d s+\int_{t_{0}}^{t} Z^{2}(s) d s
$$

has a continuous symmetric solution on $\left[t_{0}, \infty\right)$ for some $t_{0} \geq a$.

If (1.4) has a solution $y=y(t)$ with $y(t) \neq 0$ for $t \geq t_{0}$, then $r=-y^{\prime} / y$ is a solution of $(2.1)$ on $\left[t_{0}, \infty\right)$; similarly, if $Y=Y(t)$ is a nontrivial prepared solution of (1.1) with $\operatorname{det} Y(t) \neq 0$ for $t \geq t_{0}$ then $Z=-Y^{\prime} Y^{-1}$ is a symmetric solution of $(2.2)$ on $\left[t_{0}, \infty\right)$.

Hartman [11] has shown that if $(1.4)$ is nonoscillatory on $[a,+\infty)$ then a necessary and sufficient condition that

$$
\int^{\infty}\left(\frac{u^{\prime}}{u}\right)^{2} d t<\infty
$$


holds for a solution $u \not \equiv 0$ of (1.4) is that

$$
\liminf _{T \rightarrow \infty} \frac{1}{T} \int_{a}^{T} \int_{a}^{t} q(s) d s d t>-\infty
$$

One can therefore consider the cases where (2.4) does or does not hold when studying the oscillatory behavior of (1.4).

As an extension of the Fite-Wintner criterion (1.8), Olech, Opial, and Wazewski in [21] showed that $(1.4)$ is oscillatory in case

$$
\underset{T \rightarrow \infty}{\lim \operatorname{approx}} \int_{a}^{T} q(s) d s=+\infty
$$

or in case

$$
\underset{T \rightarrow \infty}{\lim \operatorname{approx} i n f} \int_{a}^{T} q(s) d s<\lim \underset{T \rightarrow \infty}{\operatorname{approx}} \sup \int_{a}^{T} q(s) d s .
$$

Wintner in [29] showed that

$$
\lim _{T \rightarrow \infty} \frac{1}{T} \int_{a}^{T} \int_{a}^{t} q(s) d s d t=+\infty
$$

implies oscillation of (1.4) and Hartman [12] showed that

$$
-\infty<\liminf _{T \rightarrow \infty} \frac{1}{T} \int_{a}^{T} \int_{a}^{t} q(s) d s d t<\limsup _{T \rightarrow \infty} \frac{1}{T} \int_{a}^{T} \int_{a}^{t} q(s) d s d t
$$

is also sufficient for oscillation of (1.4). These results were further generalized by Willett $[\mathbf{2 7}, \mathbf{2 8}]$ via more general averaging techniques.

Analogous to (2.4), we consider the (extended real-valued) function $L$ which is defined on the class of $n \times n$ continuous real symmetric matrices defined on $[a, \infty)$ by

$$
L(Q) \equiv \liminf _{T \rightarrow \infty} \frac{1}{T} \int_{a}^{T} \int_{a}^{t} \operatorname{tr} Q(s) d s d t .
$$

We now state the main results of this section.

THEOREM 2.1. Assume $L(Q)>-\infty$. Then equation (1.1) is oscillatory in case any of the following conditions hold:

$$
\begin{aligned}
& \limsup _{T \rightarrow \infty} \frac{1}{T} \int_{a}^{T} \lambda_{1}\left(\int_{a}^{t} Q(s) d s\right) d t=+\infty, \\
& \limsup _{T \rightarrow \infty} \frac{1}{T} \int_{a}^{T}\left[\lambda_{1}\left(\int_{a}^{t} Q(s) d s\right)\right]^{2} d t=+\infty \\
& \underset{T \rightarrow \infty}{\lim \operatorname{approx} \sup } \lambda_{1}\left(\int_{a}^{T} Q(s) d s\right)=+\infty, \\
& \underset{T \rightarrow \infty}{\lim \operatorname{approx} \inf } \lambda_{1}\left(\int_{a}^{T} Q(s) d s\right)=-\infty .
\end{aligned}
$$


THEOREM 2.2. Assume $L(Q)=-\infty$. Then (1.1) is oscillatory if

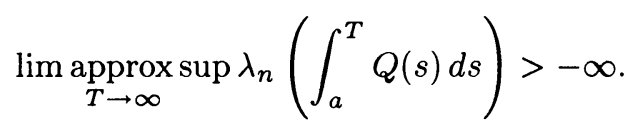

THEOREM 2.3. Assume that $\lambda_{1}\left(\int_{a}^{t} Q(s) d s\right)>0$ for sufficiently large $t$, and that

$$
\liminf _{t \rightarrow \infty}\left|\frac{\lambda_{1}\left(\int_{a}^{t} Q(s) d s\right)}{\lambda_{n}\left(\int_{a}^{t} Q(s) d s\right)}\right|>0 .
$$

Then (1.1) is oscillatory if

$$
\lim _{T \rightarrow \infty} \frac{1}{T} \int_{a}^{T} \lambda_{1}\left(\int_{a}^{t} Q(s) d s\right) d t=+\infty
$$

Since $\operatorname{tr} Q$ is a positive linear functional, systems analogues for each of the above scalar criteria (1.8), (2.5)-(2.8) may be obtained if $q(t)$ is replaced by $\operatorname{tr} Q(t)$ (see the discussion of this technique in the introduction). The intention behind Theorems 2.1 and 2.3 is to show that the corresponding (and in general, much weaker) assumptions concerning the behavior of $\lambda_{1}\left(\int_{a}^{t} Q(s) d s\right)$ will provide oscillation criteria for the system (1.1). We observe then, that Theorems 2.1(A) and 2.3 are generalizations of the results (2.8) of Hartman and a partial generalization of the result (2.7) of Wintner, respectively, and Theorems 2.1(C), (D) are partial generalizations of the oscillation criteria results (2.5), (2.6) of Olech et al. The scalar version of Theorem 2.1(B), namely that condition (2.4) and

$$
\limsup _{T \rightarrow \infty} \frac{1}{T} \int_{a}^{T}\left(\int_{a}^{t} q(s) d s\right)^{2} d t=\infty
$$

imply oscillation, although a fairly simple result, does not appear to be explicitly stated in the literature. The scalar analogue of Theorem 2.2 also does not appear to be stated explicitly elsewhere, although it is related to results of Kwong and Zettl [15].

As was mentioned in $\S 1$, the systems analogue of the Fite-Wintner theorem has been recently obtained [6]. That a complete analogue of the corresponding scalar oscillation theorem employing only the behavior of $\lambda_{1}$ is not always available for systems will be shown for the Wintner criterion (2.7) in $\S 4$.

3. Variational techniques. In this section, we turn our attention to the use of variational principles for obtaining oscillation criteria for (1.1). We assume that $Q(t)$ is symmetric and locally integrable on $[a, \infty)$. For any subinterval $[\alpha, \beta]$ of $[a, \infty)$, define $A_{1}(\alpha, \beta)$ to be

$$
\left\{\eta:[\alpha, \beta] \rightarrow \mathbf{R}^{n} \mid \eta(\alpha)=\eta(\beta)=0, \eta \in A C[\alpha, \beta], \eta^{\prime} \in L^{2}(\alpha, \beta)\right\} .
$$

The basic result we need here [12] is that (1.1) is oscillatory if and only if there is a sequence of intervals $\left[a_{n}, b_{n}\right]$, with $\lim _{n \rightarrow \infty} a_{n}=\infty$, and a sequence of functions $\varphi_{n} \in A_{1}\left(a_{n}, b_{n}\right)$, such that

$$
\int_{a_{n}}^{b_{n}}\left\{\left|\varphi_{n}^{\prime}(t)\right|^{2}-\varphi_{n}^{*}(t) Q(t) \varphi_{n}(t)\right\} d t<0
$$


The idea then is to utilize conditions on the behavior of $Q$ or its integral in order to construct sequences fulfilling (3.1).

To illustrate this technique, we present three oscillation criteria and a comparison theorem for oscillation. Theorem 3.1 is a generalization of a result of Moore [20] in the scalar case and Theorem 3.3 is a generalization of a result of Olech et al. [21] in the scalar case (see (2.8) and also Theorem 2.1(c) in §2). The comparison result, Theorem 3.4, is an extension to systems of the "telescoping" principle of Kwong and Zettl [16]. Theorem 3.2 has no scalar analogue.

THEOREM 3.1. Suppose that

$$
\limsup _{t \rightarrow \infty} \lambda_{1}\left(\int_{a}^{t} Q(s) d s\right)=\infty .
$$

Then (1.1) is oscillatory if either

$$
\text { (A) } \lambda_{1}(Q(t)) \text { is bounded above on }[a, \infty) \text {, }
$$

or

$$
\text { (B) } \lambda_{n}(Q(t)) \text { if bounded below on }[a, \infty) \text {. }
$$

THEOREM 3.2. Let the entries of $Q(t)$ be $q_{i j}(t) ; i, j=1, \ldots, n$. Assume that each $q_{i j}(t)$ is bounded on $[a, \infty)$ and $q_{i i} \equiv 0, i=1, \ldots, n$.

Then (1.1) is oscillatory if there exist $i, j$ such that

$$
\limsup _{t \rightarrow \infty} \int_{a}^{t} q_{i j}(s) d s=\infty .
$$

THEOREM 3.3. Suppose that for each positive integer $m \geq a$, there exists a positive number $\varepsilon_{m}$, and for each positive integer $k$, there exists a unit vector $x_{m k} \in$ $\mathbf{R}^{n}$ such that the set

$$
S_{m k}=\left\{t \geq m: x_{m k}^{*}\left(\int_{m}^{t} Q(s) d s\right) x_{m k} \geq k\right\}
$$

has measure at least $\varepsilon_{m}$. Then (1.1) is oscillatory.

In order to state the next result, we need to introduce classes of "telescoping" transformations discussed for the scalar case in [16]. For any (extended) real numbers $\alpha, \beta$ with $\alpha<\beta \leq \infty$ and any natural number $n$, let $D_{\alpha}^{\beta}(n)$ denote the set of piecewise continuous $n \times n$ matrix-valued functions on $[\alpha, \beta)$ and let $S=\bigcup_{i=1}^{N}\left(\alpha_{i}, \beta_{i}\right)$ be a denumerable (finite or infinite) union of subintervals of $[\alpha, \beta)$ such that

$$
\alpha<\alpha_{i}<\beta_{i}<\alpha_{i+1}<\beta \quad \text { for each } i \text {. }
$$

If $Q \in D_{\alpha}^{\beta}(n)$, then $T_{S}(Q)$ is defined to be a piecewise continuous $n \times n$ matrixvalued function $\tilde{Q}$, defined on some interval $[\alpha, A)$, which is obtained by "collapsing" each interval $\left(\alpha_{i}, \beta_{i}\right)$ to a point. Here,

$$
\begin{aligned}
A & =\sum_{i=1}^{N}\left(\alpha_{i}-\beta_{i-1}\right), \quad \text { if } \lim _{i \rightarrow N} \beta_{i}=\beta\left(\beta_{0}=\alpha\right), \\
& =\sum_{i=1}^{N}\left(\alpha_{i}-\beta_{i-1}\right)+\beta-l, \quad \text { if } \lim _{i \rightarrow N} \beta_{i}=l<\beta .
\end{aligned}
$$

We refer to [16] for further details. 
THEOREM 3.4. Let $S=\bigcup_{i=1}^{N}\left(\alpha_{i}, \beta_{i}\right)$ be a denumerable union of subintervals of $[\alpha, \infty)$ satisfying (3.4) (with $\alpha_{1}=a, \beta_{N}=\infty$ ). Assume that

$$
\int_{\alpha_{i}}^{\beta_{i}} Q(t) d t \text { is nonnegative definite for each } i \text {, }
$$

and suppose that

$$
Y^{\prime \prime}+\tilde{Q}(t) Y=0, \quad t \in[a, A)
$$

is oscillatory, where $\tilde{Q}=T_{S}(Q)$. Then (1.1) is oscillatory.

\section{Examples and counterexamples.}

EXAMPLE 1. We wish to indicate here that in Theorem 2.1, parts (A), (B), one cannot replace the average of $\lambda_{1}\left(\int_{a}^{t} Q(s) d s\right)$ or $\left(\lambda_{1}\left(\int_{a}^{t} Q(s) d s\right)^{2}\right.$ by these expressions alone, and that one cannot replace "lim approx sup" by "limsup" in parts (C), (D). It suffices to consider a scalar counterexample. We choose $v \in$ $C^{1}[a, \infty) \cap L^{2}[a, \infty)$ and define $q(t)=v^{\prime}-v^{2}$. Then $y=\exp \left(-\int_{a}^{t} v(s) d s\right)$ is a nonoscillatory solution of $y^{\prime \prime}+q y=0$ and we have

$$
\int_{a}^{t} q(s) d s=v(t)-v(a)-\int_{a}^{t} v^{2}(s) d s .
$$

It is clear that we can choose $v$ such that $\limsup _{t \rightarrow \infty} v(t)$ takes on any value $M$ with $-\infty<M \leq+\infty, \liminf _{t \rightarrow \infty} v(t)$ takes on any value $m,-\infty \leq m \leq M \leq+\infty$, and such that $\liminf _{T \rightarrow \infty}(1 / T) \int_{a}^{T} v(s) d s>-\infty$.

EXAMPLE 2. The condition

$$
\lim _{t \rightarrow \infty} \frac{1}{t} \int_{a}^{t} \lambda_{1}\left(\int_{a}^{s} Q(\sigma) d \sigma\right) d s=\infty
$$

is not by itself an oscillation criteria for (1.1) when $n>1$. To see this, consider the case where $Q(t)$ is a $2 \times 2$ diagonal matrix for all $t$, say $Q(t)=\operatorname{diag}\left\{q_{1}(t), q_{2}(t)\right\}$.

Then (1.1) uncouples as two scalar equations

$$
y_{i}^{\prime \prime}+q_{i}(t) y_{i}=0 ; \quad i=1,2 .
$$

If we take $a=0$ and denote $\int_{0}^{t} q_{i}(s) d s$ by $p_{i}(t)$, we will obtain the required counterexample if we can find $q_{1}(t), q_{2}(t)$ so that $(4.3)_{1}$ and $(4.3)_{2}$ are both nonoscillatory, with

$$
\begin{gathered}
p_{2}(t) \geq 0, \quad t \geq 0, \\
\lim _{t \rightarrow \infty} \frac{1}{t} \int_{0}^{t}\left(p_{1}(t)\right)_{+} d t=\infty, \quad \text { where }\left(p_{1}(t)\right)_{+}=\max \left(0, p_{1}(t)\right) .
\end{gathered}
$$

For then, on choosing

$$
c_{1}(t)=\left\{\begin{array}{ll}
1, & t \in \text { support }\left(p_{1}(t)\right)_{+}, \\
0, & \text { otherwise }
\end{array} \quad c_{2}(t)= \begin{cases}0, & t \in \text { support }\left(p_{1}(t)\right)_{+}, \\
1, & \text { otherwise }\end{cases}\right.
$$

and defining $x(t)=\left(c_{1}(t), c_{2}(t)\right)^{*}$, we have

$$
\begin{aligned}
\lambda_{1}\left(\int_{0}^{t} Q(t) d s\right) & \geq x^{*}(t)\left(\int_{0}^{t} Q(s) d s\right) x(t) \\
& =c_{1}^{2}(t) p_{1}(t)+c_{2}^{2}(t) p_{2}(t) \geq\left(p_{1}(t)\right)_{+} .
\end{aligned}
$$


Thus (4.2) holds, but clearly (1.1) is nonoscillatory. The choice of $q_{2}(t)$ is straightforward, e.g. take $q_{2}(t)=1 / 4(1+t)^{2}$. To construct a suitable $q_{1}(t)$, we first give an inductive definition of a suitable Riccati scalar variable $z_{1}(t)$. Define $z_{1}$ on $[0,1]$ by $z_{1}(0)=0, z_{1}(t)=1, \frac{1}{2} \leq t \leq 1$, so that $z_{1}$ is continuously differentiable and monotone nondecreasing on $[0,1]$. Define $a_{1}$ to be $\int_{0}^{1} z_{1}^{2}(s) d s, b_{1}$ to be $4 a_{1}$ and $\varepsilon_{1}$ to be $1 / 8 b_{1}$. Then $a_{1} \geq \frac{1}{2}, b_{1} \geq 2$ and $2 \varepsilon_{1} \leq \frac{1}{8}<\frac{1}{2}$. Let $s_{m}=\sum_{i=1}^{m} 1 / i$. Extend $z_{1}$ to $\left[s_{1}, s_{2}\right]$ by taking $z_{1}(t)=b_{1}$ on $\left[1+\varepsilon_{1}, s_{2}\right]$ and making $z_{1}$ continuously differentiable and monotone nondecreasing on $\left[0, s_{2}\right]$. Inductively, suppose that $a_{i}, b_{i}, \varepsilon_{i}$ have been defined for $1 \leq i \leq m$, with $b_{i}=4 a_{i}, \varepsilon_{i}=1 / 8 b_{i}<1 / 2(i+1)$, and $z_{1}$ has been defined as a continuously differentiable, monotone nondecreasing function on $\left[0, s_{m+1}\right]$ such that $z_{1}(t) \equiv b_{i}$ on $\left[s_{i}+\varepsilon_{i}, s_{i+1}\right]$ and $\int_{0}^{s_{i}} z_{1}^{2}(s) d s=a_{i}, 1 \leq i \leq m$. Then

$$
a_{m+1} \geq a_{m}+\int_{s_{m}+\varepsilon_{m}}^{s_{m}+2 \varepsilon_{m}} z_{1}^{2}(s) d s=a_{m}+b_{m}^{2} \varepsilon_{m} \geq \frac{3}{2} a_{m} .
$$

By the inductive hypothesis, we see that $a_{m+1} \geq(3 / 2)^{m} a_{1} \geq \frac{1}{2}(3 / 2)^{m}$. If $b_{m+1}=$ $4 a_{m+1}, \varepsilon_{m+1}=1 / 8 b_{m+1}$, then $\varepsilon_{m+1} \leq(3 / 2)^{-m} / 16 \leq 1 / 2(m+2)$ (since $(3 / 2)^{m}=$ $\left.(1+1 / 2)^{m} \geq 1+m / 2>2(m+2) / 16\right)$, and we extend $z_{1}$ to $\left[s_{m+1}, s_{m+2}\right]$ so that $z_{1}(t) \equiv b_{m+1}$ on $\left[s_{m+1}+\varepsilon_{m+1}, s_{m+2}\right]$ and is continuously differentiable and monotone nondecreasing on $\left[0, s_{m+2}\right]$. With this inductive definition of $z_{1}$ on $[0, \infty)$, note that if $t \in\left[s_{m}+\varepsilon_{m}, s_{m}+2 \varepsilon_{m}\right]$, we have

$$
z_{1}(t)-\int_{0}^{t} z_{1}^{2}(s) d s \geq b_{m}-a_{m}-2 \varepsilon_{m} b_{m}^{2} \geq \frac{1}{2} b_{m} .
$$

Define $q_{1}(t)$ to be $z_{1}^{\prime}(t)-z_{1}^{2}(t)$. Then for $t \in\left[s_{k}+\varepsilon_{k}, s_{k}+2 \varepsilon_{k}\right]$, we have

$$
p_{1}(t)=\int_{0}^{t} q_{1}(s) d s \geq \frac{1}{2} b_{k} .
$$

Thus if $s_{m} \leq t \leq s_{m+1}$, we have

$$
\frac{1}{t} \int_{0}^{t}\left(p_{1}(s)\right)_{+} d s \geq \frac{1}{t} \sum_{k=1}^{m} \frac{1}{2} b_{k} \cdot \varepsilon_{k}=\frac{m}{16 t} .
$$

Since $s_{m} \sim \log m$, for $s_{m} \leq t \leq s_{m+1}$ we have $m \sim e^{t}$ so that

$$
\frac{1}{t} \int_{0}^{t}\left(p_{1}(s)\right)_{+} d s \sim \frac{e^{t}}{16 t}
$$

Thus (4.5) holds, yet (4.3) ${ }_{1}$ is nonoscillatory, and so the example is complete.

EXAMPLE 3. Akiyama [1] conjectured that a condition weaker than (1.10), namely

$$
\lim _{t \rightarrow \infty} \int_{0}^{t} \lambda_{1}(Q(s)) d s=\infty
$$

might suffice for oscillation of (1.1).

The previous example may be used to provide a counterexample to this conjecture. For if we define $q_{1}(t), q_{2}(t), z_{1}(t)$, etc. as in that example, then

$$
\int_{0}^{t} \lambda_{1}(Q(s)) d s \geq \int_{0}^{t}\left(q_{1}(s)\right)_{+} d s=\int_{0}^{t}\left(z_{1}^{\prime}(s)-z_{1}^{2}(s)\right)_{+} d s .
$$


Now on $\left[s_{m}, s_{m}+\varepsilon_{m}\right]$, we have $z_{1}(s) \leq b_{m}$, and so

$$
\begin{aligned}
\int_{s_{m}}^{s_{m}+\varepsilon_{m}}\left(z_{1}^{\prime}(s)\right. & \left.-z_{1}^{2}(s)\right)_{+} d s \geq \int_{s_{m}}^{s_{m}+\varepsilon_{m}}\left(z_{1}^{\prime}(s)-z_{1}^{2}(s)\right) d s \\
& =z_{1}\left(s_{m}+\varepsilon_{m}\right)-z_{1}\left(s_{m}\right)-\int_{s_{m}}^{s_{m}+\varepsilon} z_{1}^{2}(s) d s \\
& \geq b_{m}-b_{m-1}-b_{m}^{2} \varepsilon_{m} \geq 4\left(a_{m}-a_{m-1}\right)-\frac{1}{8} b_{m} \\
& =\frac{4}{3} a_{m}-\frac{1}{8} b_{m}=\frac{5}{24} b_{m},
\end{aligned}
$$

and so

$$
\int_{0}^{s_{m+1}} \lambda_{1}(Q(s)) d s \geq \frac{5}{24} \sum_{k=1}^{m} b_{k} \rightarrow \infty \quad \text { as } m \rightarrow \infty .
$$

5. Proofs for §2. The proofs of Theorem 2.1 and Theorem 2.2 will make use of the following lemma which is the systems analogue of the result of Hartman mentioned in $\S 2$ (cf. (2.3), (2.4)).

LeMMA 5.1. Assume equation (1.1) is nonoscillatory on $[a,+\infty)$. Then a necessary and sufficient condition that

$$
\lim _{T \rightarrow \infty} \int_{t}^{T} Z^{2}(s) d s
$$

exists for any solution $Z=-Y^{\prime} Y^{-1}$ of (2.2) where $Y(t)$ is a prepared solution of (1.1), is that

$$
L(Q)=\liminf _{T \rightarrow \infty} \frac{1}{T} \int_{a}^{T} \int_{a}^{t} \operatorname{tr} Q(s) d s d t>-\infty .
$$

Proof. Suppose first that the limit in (5.1) exists for some solution $Z=$ $-Y^{\prime} Y^{-1}$ of $(2.2)$, where $\operatorname{det} Y(t) \neq 0, t \geq a$, so that we have from (2.2)

$$
\operatorname{tr} Z(t)+\operatorname{tr} \int_{t}^{\infty} Z^{2}(s) d s=\int_{a}^{t} \operatorname{tr} Q(s) d s-C,
$$

where $C=-\operatorname{tr} Z(a)-\operatorname{tr} \int_{a}^{\infty} Z^{2}(s) d s$. Since

$$
\begin{aligned}
& \frac{1}{T} \int_{a}^{T}\left(\operatorname{tr} Z(t)-\int_{t}^{\infty} \operatorname{tr} Z^{2}(s) d s\right)^{2} d t \\
& \quad \leq \frac{2}{T}\left[\int_{a}^{T}\left((\operatorname{tr} Z(t))^{2}+\left(\int_{t}^{\infty} \operatorname{tr} Z^{2}(s) d s\right)^{2}\right) d t\right]
\end{aligned}
$$

and since $\operatorname{tr} Z(t)^{2} \leq n \operatorname{tr} Z^{2}(t)$, we have $(1 / T) \int_{a}^{T} \operatorname{tr} Z^{2}(t) \rightarrow 0$ as $T \rightarrow \infty$. Similarly, $(1 / T) \int_{a}^{T}\left(\int_{t}^{\infty} \operatorname{tr} Z^{2}(s) d s\right)^{2} d t \rightarrow 0$ as $T \rightarrow \infty$ so that (5.3) and (5.4) imply

$$
\frac{1}{T} \int_{a}^{T}\left[\int_{a}^{t} \operatorname{tr} Q(s) d s-C\right]^{2} d t \rightarrow 0 \quad \text { as } T \rightarrow \infty
$$


Now

$$
\left|\frac{1}{T} \int_{a}^{T}\left[\int_{a}^{t} \operatorname{tr} Q(s) d s-C\right] d t\right| \leq\left(\frac{1}{T} \int_{a}^{T}\left[\int_{a}^{t} \operatorname{tr} Q(s) d s-C\right]^{2} d t\right)^{1 / 2}
$$

by the Cauchy-Schwarz inequality and therefore it follows that

$$
\lim _{T \rightarrow \infty} \frac{1}{T} \int_{a}^{T}\left[\int_{a}^{t} \operatorname{tr} Q(s) d s\right] d t=C \quad \text { exists }
$$

so that (5.2) holds.

Conversely, suppose (5.2) holds and let $Z=-Y^{\prime} Y^{-1}$, where $Y(t)$ is a prepared solution of $(1.1)$ with $\operatorname{det} Y(t) \neq 0, t \geq a$. From (2.2) we have

$$
\begin{aligned}
-\frac{1}{T} \int_{a}^{T} Z(t) d t & +\frac{1}{T} \int_{a}^{T} \int_{a}^{t} Z^{2}(s) d s d t \\
& =-\frac{1}{T}(T-a) Z(a)-\frac{1}{T} \int_{a}^{T} \int_{a}^{t} Q(s) d s d t
\end{aligned}
$$

so that from (5.2) we have

$$
-\frac{1}{T} \int_{a}^{T} \operatorname{tr} Z(s) d s+\frac{1}{T} \int_{a}^{T} \int_{a}^{t} \operatorname{tr} Z^{2}(s) d s d t \leq M, \quad \text { for some } M>0 .
$$

Since $\operatorname{tr} Z^{2}(t) \geq 0$, it follows that $\lim _{t \rightarrow \infty} \int_{a}^{t} \operatorname{tr} Z^{2}(s) d s$ exists, finite or infinite.

Suppose that $\int_{a}^{t} \operatorname{tr} Z^{2}(s) d s \rightarrow+\infty$ as $t \rightarrow+\infty$. Then

$$
\frac{1}{T} \int_{a}^{T} \int_{a}^{t} \operatorname{tr} Z^{2}(s) d s d t \rightarrow \infty \quad \text { as } T \rightarrow+\infty
$$

From (5.8), it follows that $(1 / T) \int_{a}^{T} \operatorname{tr} Z(s) d s \rightarrow \infty$ as $T \rightarrow \infty$, and so for large $T$ we have, again using (5.8),

$$
\frac{1}{T} \int_{a}^{T} \int_{a}^{t} \operatorname{tr} Z^{2}(s) d s d t \leq \frac{1}{T} \int_{a}^{T} \operatorname{tr} Z(s) d s+M \leq \frac{2}{T} \int_{a}^{T} \operatorname{tr} Z(s) d s .
$$

Now by the Cauchy-Schwarz inequality we have

$$
\left|\frac{1}{T} \int_{a}^{T} \operatorname{tr} Z(s) d s\right| \leq\left(\frac{1}{T} \int_{a}^{T}(\operatorname{tr} Z(s))^{2} d s\right)^{1 / 2} \leq\left(\frac{n}{T} \int_{a}^{T} \operatorname{tr} Z^{2}(s) d s\right)^{1 / 2}
$$

so that (5.9) gives

$$
\left(\frac{1}{T} \int_{a}^{T} \int_{a}^{t} \operatorname{tr} Z^{2}(s) d s d t\right)^{2} \leq \frac{4 n}{T} \int_{a}^{T} \operatorname{tr} Z^{2}(s) d s .
$$

If we set $H(T) \equiv \int_{a}^{T} \int_{a}^{t} \operatorname{tr} Z^{2}(s) d s d t$, we have from (5.10) that

$$
H^{2}(t) \leq 4 n t H^{\prime}(t), \quad t \geq T_{1},
$$

and so

$$
\frac{1}{4 n t} \leq \frac{H^{\prime}(t)}{H^{2}(t)}, \quad t \geq T_{1}
$$


Now an integration of $(5.12)$ over $\left[T_{1}, \infty\right)$ gives

$$
\left.\infty=\frac{1}{4 n} \ln t\right]_{T_{1}}^{\infty} \leq \frac{1}{H\left(T_{1}\right)}<+\infty
$$

a contradiction. Thus $\lim _{t \rightarrow \infty} \int_{a}^{t} \operatorname{tr} Z^{2}(s) d s$ exists as a finite limit.

We see that this implies the existence of $\lim _{t \rightarrow \infty} \int_{a}^{t} Z^{2}(s) d s$, as follows: let the (operator) norm of a matrix $A$ be denoted by $|A|$. For $a \leq s \leq t$, define $A(s, t)$ by $A(s, t)=\int_{a}^{t} Z^{2}(\sigma) d \sigma$. Then $A(s, t)$ is a nonnegative definite matrix and $|A(s, t)|^{a}=\lambda_{1}(A(s, t)) \leq \operatorname{tr} A(s, t)=\int_{s}^{t} \operatorname{tr} Z^{2}(\sigma) d \sigma$. This last integral converges to zero as $s, t \rightarrow \infty$ and so we have $|A(s, t)| \rightarrow 0$ as $s, t \rightarrow \infty$, i.e. $\int_{s}^{t} Z^{2}(\sigma) d \sigma \rightarrow 0$ as $s, t \rightarrow \infty$, yielding the existence of $\lim _{t \rightarrow \infty} \int_{a}^{t} Z^{2}(s) d s$ as asserted. This completes the proof of the lemma.

Proof of ThEOREM 2.1. (A) Assume $L(Q)>-\infty$ and that

$$
\limsup _{T \rightarrow \infty} \frac{1}{T} \int_{a}^{T} \lambda_{1}\left(\int_{a}^{t} Q(s) d s\right) d t=+\infty
$$

Suppose there exists a prepared solution $Y(t)$ of $(1.1)$ which is not oscillatory. Without loss of generality, we may suppose that $\operatorname{det} Y(t) \neq 0, t \geq a$, so that from (2.2) we have (with $Z=-Y^{\prime} Y^{-1}$ )

$$
\lambda_{1}(Z(t)-Z(a))=\lambda_{1}\left(\int_{a}^{t} Q(s) d s+\int_{a}^{t} Z^{2}(s) d s\right) .
$$

By the convexity of $\lambda_{1}$ and the fact that $\int_{a}^{t} Z^{2}(s) d s \geq 0$ (i.e., nonnegative definite for $t \geq a)$, we have from (5.14) that $\lambda_{1}\left(Z(t)+\lambda_{1}(-Z(a)) \geq \lambda_{1}\left(\int_{a}^{t} Q(s) d s\right)\right.$ and hence

$$
\frac{1}{T} \int_{a}^{T} \lambda_{1}(Z(s)) d s+\frac{T-a}{T} \lambda_{1}(-Z(a)) \geq \frac{1}{T} \int_{a}^{T} \lambda_{1}\left(\int_{a}^{t} Q(s) d s\right) d t
$$

so that from hypothesis (A), there exists a sequence $T_{n} \rightarrow \infty$ with

$$
\frac{1}{T_{n}} \int_{a}^{T_{n}} \lambda_{1}(Z(s)) d s \rightarrow+\infty \quad \text { as } T_{n} \rightarrow \infty .
$$

Since $\lambda_{1}(Z(s))^{2} \leq \lambda_{1}\left(Z^{2}(s)\right)$ we have, by the Cauchy-Schwarz inequality

$$
\begin{aligned}
\left|\frac{1}{T_{n}} \int_{a}^{T_{n}} \lambda_{1}(Z(s)) d s\right| & \leq\left(\frac{1}{T_{n}} \int_{a}^{T_{n}}\left(\lambda_{1}(Z(s))\right)^{2} d s\right)^{1 / 2} \\
& \leq\left(\frac{1}{T_{n}} \int_{a}^{T_{n}} \lambda_{1}\left(Z^{2}(s)\right) d s\right)^{1 / 2} \rightarrow+\infty
\end{aligned}
$$

as $T_{n} \rightarrow+\infty$. But from Lemma 5.1, since $L(Q)>-\infty$ it follows that $\int_{a}^{\infty} \lambda_{1}\left(Z^{2}(s)\right) d s \leq \int_{a}^{\infty} \operatorname{tr} Z^{2}(s) d s<+\infty$, so this contradiction proves part (A).

(B) Assume $L(Q)>-\infty$ and that

$$
\lim \sup \frac{1}{T} \int_{a}^{T}\left[\lambda_{1}\left(\int_{a}^{t} Q(s) d s\right)\right]^{2} d t=+\infty
$$


As in part (A) above, we may assume $Z(t)=-Y^{\prime} Y^{-1}$ is a solution of (2.2) for $t \geq a$ and so by Lemma 5.1 , we obtain

$$
Z(t)+\int_{t}^{\infty} Z^{2}(s) d s+C=\int_{a}^{t} Q(s) d s,
$$

where $C=-Z(a)-\int_{a}^{\infty} Z^{2}(s) d s$. Hence, we have

$$
\begin{aligned}
\left(\lambda_{1}\left(\int_{a}^{t} Q(s) d s\right)\right)^{2}= & \left(\lambda_{1}\left[Z(t)+\int_{t}^{\infty} Z^{2}(s) d s+C\right]\right)^{2} \\
\leq & \lambda_{1}\left(Z(t)+\int_{t}^{\infty} Z^{2}(s) d s+C\right)^{2} \\
\leq & \operatorname{tr}\left(Z(t)+\int_{t}^{\infty} Z^{2}(s) d s+C\right)^{2} \\
\leq & 2 \operatorname{tr}\left(Z(t)+\int_{t}^{\infty} Z^{2}(s)\right)^{2}+2 \operatorname{tr} C^{2} \leq 4 \operatorname{tr} Z^{2}(t) \\
& +4 \operatorname{tr}\left(\int_{t}^{\infty} Z^{2}(s) d s\right)^{2}+2 \operatorname{tr} C^{2},
\end{aligned}
$$

(since $\operatorname{tr}(A+B)^{2} \leq 2\left(\operatorname{tr} A^{2}+\operatorname{tr} B^{2}\right)$. Therefore, we get

$$
\begin{aligned}
\frac{1}{T} \int_{a}^{T}\left[\lambda_{1}\left(\int_{a}^{t} Q(s) d s\right)\right]^{2} d t \leq & \frac{4}{T} \int_{a}^{T} \operatorname{tr}\left(\int_{t}^{\infty} Z^{2}(s) d s\right)^{2} d t \\
& +\frac{4}{T} \int_{a}^{T} \operatorname{tr} Z^{2}(t) d t+\frac{2(T-a)}{T} \operatorname{tr} C^{2} .
\end{aligned}
$$

If we set $B(t)=\int_{t}^{\infty} Z^{2}(s) d s$, then $\operatorname{tr} B(t) \rightarrow 0$ and $B(t) \geq 0$ so that $\lambda_{1}(B(t)) \rightarrow 0$ and hence $\lambda_{1}\left(B^{2}(t)\right) \rightarrow 0$. Therefore, $\operatorname{tr} B^{2}(t) \rightarrow 0$ as $t \rightarrow \infty$. Thus, the first and second integral on the right side of (5.20) tend to 0 as $T \rightarrow+\infty$ and the last term is bounded. However, condition (B) implies that the left side of (5.20) is not bounded and this contradiction completes the proof of part (B).

(C) Assume $L(Q)>-\infty$ and $\lim$ approx $\sup _{T \rightarrow \infty} \lambda_{1}\left(\int_{a}^{T} Q(s) d s\right)=+\infty$. As in part (B), we obtain equation (5.18) so that

$$
\lambda_{1}(Z(t))+\int_{t}^{\infty} \lambda_{1}\left(Z^{2}(s)\right) d s \geq \lambda_{1}\left(\int_{a}^{t} Q(s) d s\right)+\lambda_{n}(-C) .
$$

Since $\int_{t}^{\infty} \lambda_{1}\left(Z^{2}(s)\right) d s \rightarrow 0$ as $t \rightarrow \infty$, it follows that $\int_{t}^{\infty}\left[\lambda_{1}(Z(s))\right]^{2} d s \rightarrow 0$ as $t \rightarrow \infty$ and $\lambda_{1}\left(\int_{t}^{\infty} Z^{2}(s) d s\right) \rightarrow 0$ as $t \rightarrow \infty$. Now for any $k \geq 1$,

$$
\mu\left\{t: \lambda_{1}\left(\int_{a}^{t} Q(s) d s\right) \geq k\right\}=+\infty
$$

so that if $k \geq\left|\lambda_{n}(-C)\right|+1$, then from (5.21)

$$
\mu\left\{t: \lambda_{1}(Z(t))+\lambda_{1}\left(\int_{t}^{\infty} Z^{2}(s) d s\right) \geq 1\right\}=+\infty .
$$

Since $\lambda_{1}\left(\int_{t}^{\infty} Z^{2}(s) d s\right)<\frac{1}{2}$ if $t \geq T_{0}$, say, we see that

$$
\mu\left\{t: \lambda_{1}(Z(t)) \geq \frac{1}{2}\right\}=+\infty .
$$


That is, $\int_{E_{k}}\left(\lambda_{1}(Z(t))\right)^{2} d t=+\infty$, where $E_{k}=\left\{t: \lambda_{1}(Z(t)) \geq \frac{1}{2}\right\}$. This contradiction proves part $(\mathrm{C})$.

(D) Assume $L(Q)>-\infty$ and lim approxinf ${ }_{T \rightarrow \infty} \lambda_{1}\left(\int_{a}^{T} Q(s) d s\right)=-\infty$. The proof is similar to part (C). Since for any $M>0$,

$$
\mu\left\{t: \lambda_{1}\left(\int_{a}^{t} Q(s) d s\right)<-M\right\}=+\infty
$$

and since

$$
\lambda_{1}(Z(t)) \leq \lambda_{1}\left(Z(t)+\int_{t}^{\infty} Z^{2}(s) d s\right) \leq \lambda_{1}\left(\int_{a}^{t} Q(s) d s\right)+\lambda_{1}(-C)
$$

it follows that if $M>1+\left|\lambda_{1}(-C)\right|$, we have $\mu\left\{t: \lambda_{1}(Z(t)) \leq-1\right\}=+\infty$ so that $\int_{a}^{\infty}\left(\lambda_{1}(Z(t))\right)^{2} d t=+\infty$, a contradiction. This completes the proof of Theorem 2.1.

PROOF OF THEOREM 2.2. Assume $L(Q)=-\infty$ and

$$
\lim \underset{t \rightarrow \infty}{\operatorname{approx} \sup } \lambda_{n}\left(\int_{a}^{t} Q d s\right)=m>-\infty
$$

If the equation is nonoscillatory, then without loss of generality there is a solution $Y$ of $(1.1)$ for which $\operatorname{det} Y(t) \neq 0$ on $[a, \infty)$. Then with $Z=-Y^{\prime} Y^{-1}, t \geq a$, we get

$$
Z(t)=Z(a)+\int_{a}^{t} Z^{2}(s) d s+\int_{a}^{t} Q(s) d s
$$

By Lemma 5.1, since $L(Q)=-\infty$, it follows that $\operatorname{tr} \int_{a}^{t} Z^{2}(s) d s \rightarrow+\infty$ as $t \rightarrow$ $+\infty$ and hence $\lambda_{1}\left(\int_{a}^{t} Z^{2}(s) d s\right) \rightarrow+\infty$ as $t \rightarrow+\infty$. Since $\lambda_{1}\left(-\int_{a}^{t} Q(s) d s\right)=$ $-\lambda_{n}\left(\int_{a}^{t} Q(s) d s\right)$ we have from (5.26)

$$
\begin{aligned}
\lambda_{1}\left(Z(t)-\int_{a}^{t} Q(s) d s\right) & \leq \lambda_{1}(Z(t))+\lambda_{1}\left(-\int_{a}^{t} Q(s) d s\right) \\
& =\lambda_{1}(Z(t))-\lambda_{n}\left(\int_{a}^{t} Q(s) d s\right) .
\end{aligned}
$$

Now for any $\varepsilon>0, \mu\left\{t: \lambda_{n}\left(\int_{a}^{t} Q(s) d s\right) \geq m-\varepsilon\right\}=+\infty$. From (5.26) we have

$$
\begin{aligned}
\frac{1}{n} \operatorname{tr} \int_{a}^{t} Z^{2}(s) d s & =\frac{1}{n} \operatorname{tr}\left(Z(t)-\int_{a}^{t} Q(s) d s-Z(a)\right) \\
& =\frac{1}{n} \operatorname{tr}\left(Z(t)-\int_{a}^{t} Q(s) d s\right)-\frac{1}{n} \operatorname{tr} Z(a) \\
& \leq \lambda_{1}\left(Z(t)-\int_{a}^{t} Q(s) d s\right)-\frac{1}{n} \operatorname{tr} Z(a) \\
& \leq \lambda_{1}(Z(t))+\lambda_{1}\left(-\int_{a}^{t} Q(s) d s\right)-\frac{1}{n} \operatorname{tr} Z(a) \\
& =\lambda_{1}(Z(t))-\lambda_{n}\left(\int_{a}^{t} Q(s) d s\right)-\frac{1}{n} \operatorname{tr} Z(a)
\end{aligned}
$$


and since $(1 / n) \operatorname{tr} \int_{a}^{t} Z^{2}(s) d s \geq(1 / n) \int_{a}^{t} \lambda_{1}\left(Z^{2}(s)\right) d s$ we have from (5.28)

$$
\frac{1}{n} \operatorname{tr} Z(a)+\frac{1}{n} \int_{a}^{t} \lambda_{1}\left(Z^{2}(s)\right) d s \leq \lambda_{1}(Z(t))-\lambda_{n}\left(\int_{a}^{t} Q(s) d s\right), \quad t \geq a
$$

Hence, we have that for any $\varepsilon>0$

$$
\mu\left\{t: \frac{1}{n} \operatorname{tr} Z(a)+\frac{1}{n} \int_{a}^{t} \lambda_{1}\left(Z^{2}(s)\right) d s \leq \lambda_{1}(Z(t))-m+\varepsilon\right\}=+\infty
$$

and since $\left.\int_{a}^{t} \lambda_{1}\left(Z^{2}(s)\right) d s \geq \lambda_{1}\left(\int_{a}^{t} Z^{2}(s)\right) d s\right) \rightarrow \infty$ as $t \rightarrow+\infty$, we see that if $E$ is defined by

$$
E=\left\{t: \frac{1}{2 n} \int_{a}^{t} \lambda_{1}\left(Z^{2}(s)\right) d s \leq \lambda_{1}(Z(t))\right\} \cap[a+1, \infty)
$$

then $\mu(E)=+\infty$. But now with $P(t) \equiv \int_{a}^{t} \lambda_{1}\left(Z^{2}(s)\right) d s$, we have $P^{\prime}(t)=\lambda_{1}\left(Z^{2}(t)\right)$ $\geq \lambda_{1}(Z(t))^{2}$ and so $P^{\prime}(t) \geq P^{2}(t) / 4 n^{2}, t \in E$, and now $\int_{E} P^{\prime}(t) / P^{2}(t) d t \geq$ $\mu(E) / 4 n^{2}=+\infty$, a contradiction since the integral on the left is $\leq 1 / P(a+1)$. This completes the proof of Theorem 2.2.

Before proving Theorem 2.3, we require a lemma.

LEMMA 5.2. Let $p(t)$ be locally bounded, nonnegative and measurable on $[a, \infty)$ with $p(t)$ not almost everywhere zero. Let $q(t)$ be nonnegative and locally integrable such that

$$
\int_{a}^{t} p(s) d s \geq q(t) \int_{a}^{t} \int_{a}^{s} p^{2}(\sigma) d \sigma d s, \quad \text { for almost all } t \geq a .
$$

Then for all sufficiently large $\bar{a}>a, t^{-1 / 2} q(t) \in L^{2}[\bar{a}, \infty)$.

ProOF. Let $P(t)=\int_{a}^{t} \int_{a}^{S} p^{2}(\sigma) d \sigma d s$. Then $P$ is continuously differentiable and $P(t)>0$ for $t>a^{*}$, say. We have $P^{\prime}(t)=\int_{a}^{t} p^{2}(s) d s$ and by the Cauchy-Schwarz inequality

$$
P^{\prime}(t) \geq(t-a)^{-1}\left(\int_{a}^{t} p(s) d s\right)^{2} \geq(t-a)^{-1} q^{2}(t) P^{2}(t) .
$$

Since $\int_{\bar{a}}^{\infty} P^{\prime}(t) / P^{2}(t) d t<\infty$ for $\bar{a}>a^{*}$, the result follows.

PROOF OF THEOREM 2.3. The argument is similar to that given in the proof of Theorem 3.1 of [4], so we content ourselves here with giving a sketch of the proof. If $(1.1)$ is nonoscillatory, we may, without loss of generality, find a prepared solution $Y$ with $\operatorname{det} Y(t) \neq 0$ on $[a, \infty)$. If $Z=-Y^{\prime} Y^{-1}$, then $Z$ is symmetric and we have

$$
Z(t)=\int_{a}^{t} Z^{2}(s) d s=\int_{a}^{t} Q(s) d s+C, \quad t \geq a,
$$

for some constant matrix $C$.

It is known that for any continuous $n \times n$ symmetric matrix-valued function, a continuously varying orthonormal system may be selected [22]. Consequently, we may choose a locally integrable vector function $x(t)$ with $\|x(t)\|=1$ such that

$$
x^{*}(t)\left(\int_{a}^{t} Q(s) d s\right) x(t)=\lambda_{1}\left(\int_{a}^{t} Q(s) d s\right)
$$


(possible discontinuities in $x(t)$ arise because of the coalescence of eigenvalues).

Let the eigenvalues of $Z(t)$ be $\mu_{1}(t) \geq \cdots \geq \mu_{n}(t)$. By the preceding remark, we may select a system of orthonormal locally integrable eigenvectors $e_{i}(t)$ such that

$$
Z(t) e_{i}(t)=\mu_{i}(t) e_{i}(t) ; \quad e_{i}^{*}(t) e_{j}(t)=\delta_{i j} .
$$

Let $U(t)$ be the orthogonal matrix whose rows are the $e_{i}(t)$, and let $c_{i}(s, t)$ be the projection of $x(t)$ onto $e_{i}(s)$. Let the components of $U^{-1}(t) c(a, t)$ be $v_{i}(t)$, and let the components of $\left(U^{-1}(s)-U^{-1}(t)\right) c(a, t)$ be $w_{i}(s, t), i=1, \ldots, n$. Then if we denote the left-hand side of $(5.33)$ by $\Phi(t)$, we have (see [4])

$$
\begin{aligned}
& \lambda_{1}\left(\int_{a}^{t} Q(s) d s\right)+x^{*}(t) C x(t)=x^{*}(t) \Phi(t) x(t) \\
& \quad=\sum_{i=1}^{n} \mu_{i}(t) v_{i}^{2}(t)-\int_{a}^{t} \sum_{i=1}^{n} \mu_{i}^{2}(s)\left(v_{i}(t)+w_{i}(s, t)\right)^{2} d s .
\end{aligned}
$$

The arguments given in [4] show that hypothesis (2.7) allows us to find a unit vector $\hat{x}(t)$, functions $\hat{\mu}_{i j}(t) ; i=1, \ldots, n, j=1, \ldots, m$, and $\delta>0$ such that

$$
\begin{aligned}
\frac{1}{3} \lambda_{1}\left(\int_{a}^{t} Q(s) d s\right)-2\|C\| & \leq \hat{x}^{*}(t) \Phi(t) \hat{x}(t) \\
& \leq \sum_{i j}\left(\hat{\mu}_{i j}(t)-\delta \int_{a}^{t} \hat{\mu}_{i j}(s) d s\right) .
\end{aligned}
$$

Hypothesis (A) and (5.37) imply that there exists $t_{1}>a$ such that

$$
\int_{a}^{t} \sum_{i, j}\left(\hat{\mu}_{i j}(s)-\delta \int_{a}^{s} \hat{\mu}_{i j}^{2}(\sigma) d \sigma\right) d s>0, \quad t \geq t_{1} .
$$

If $\hat{\mu}_{i j} \equiv 0$ a.e. for $t \geq a$, let $\hat{q}_{i j}(t)=0$; otherwise define $\hat{q}_{i j}(t)$ by

$$
\int_{a}^{t}\left|\hat{\mu}_{i j}(s)\right| d s=\hat{q}_{i j}(t) \int_{a}^{t} \int_{a}^{s}\left|\mu_{i j}\right|^{2}(\sigma) d \sigma d s
$$

By Lemma 5.2, each $\hat{q}_{i j}$ has the property that $t^{-1 / 2} \hat{q}_{i j}(t) \in L^{2}[\bar{a}, \infty)$ if $\bar{a}>t_{1}$ is sufficiently large, and so $\hat{q}(t)=\sum_{i, j} \hat{q}_{i j}(t) \in L^{2}[\bar{a}, \infty)$, if $\bar{a}$ is sufficiently large. However, (5.38) implies that

$$
(\hat{q}(t)-\delta) \sum_{i, j} \int_{a}^{t} \int_{a}^{s} \hat{\mu}_{i j}(\sigma) d \sigma d s>0, \quad t>\bar{a},
$$

and so $\hat{q}(t)>\delta$ for $t>\bar{a}$, contradicting the square-integrability of $t^{-1 / 2} \hat{q}(t)$. This proves the theorem.

6. Proofs for $\S 3$.

PROOF OF THEOREM 3.1. Choose any sequence $a_{k}>a$ with $\lim _{k \rightarrow \infty} a_{k}=\infty$. We have

$$
\lambda_{1}\left(\int_{a_{k}}^{t} Q(s) d s\right) \geq \lambda_{1}\left(\int_{a}^{t} Q(s) d s\right)-\lambda_{1}\left(\int_{a}^{a_{k}} Q(s) d s\right)
$$


and so, by (3.2), $\lim \sup _{t \rightarrow \infty} \lambda_{1}\left(\int_{a_{k}}^{t} Q(s) d s\right)=\infty$. Therefore we may choose $b_{k}>$ $a_{k}+2$ and $x_{k} \in \mathbf{R}^{n}$ with $\left\|x_{k}\right\|=1$, so that

$$
x_{k}^{*}\left(\int_{a_{k}}^{b_{k}} Q(s) d s\right) x_{k} \geq k, \quad k=1,2, \ldots
$$

Suppose that condition (A) holds. Then there exists $M$ such that $\lambda_{1}(Q(t)) \leq M$, $t \geq a$. Define $\varphi_{k} \in A_{1}\left(a_{k}, b_{k}\right)$ as follows:

$$
\varphi_{k}(t)= \begin{cases}\left(t-a_{k}\right) x_{k}, & a_{k} \leq t \leq a_{k}+1, \\ x_{k}, & a_{k}+1 \leq t \leq b_{k}-1, \\ \left(-t+b_{k}\right) x_{k}, & b_{k}-1 \leq t \leq b_{k} .\end{cases}
$$

We have $\int_{a_{k}}^{b_{k}}\left|\varphi_{k}^{\prime}(t)\right|^{2} d t=2$, and

$$
\begin{aligned}
\int_{a_{k}}^{b_{k}} \varphi_{k}^{*}(t) Q(t) \varphi_{k}(t) d t= & \int_{a_{k}}^{b_{k}} x_{k}^{*} Q(t) x_{k} d t-\int_{a_{k}}^{a_{k}+1}\left[1-\left(t-a_{k}\right)^{2}\right] x_{k}^{*} Q(t) x_{k} d t \\
& -\int_{b_{k}-1}^{b_{k}}\left[1-\left(b_{k}-t\right)^{2}\right] x_{k}^{*} Q(t) x_{k} d t \geq k-\frac{4}{3} M .
\end{aligned}
$$

Thus

$$
\int_{a_{k}}^{b_{k}}\left\{\left|\varphi_{k}^{\prime}(t)\right|^{2}-\varphi_{k}^{*}(t) Q(t) \varphi_{k}(t)\right\} d t \leq 2+\frac{4}{3} M-k<0
$$

for $k$ sufficiently large. It follows that $(1.1)$ is oscillatory.

If condition (B) holds, we have $\lambda_{n}(Q(t)) \geq-M, t \geq a$, and we define $\varphi_{k} \in$ $A_{1}\left(a_{k}-1, b_{k}+1\right)$ by

$$
\varphi_{k}(t)= \begin{cases}\left(t+1-a_{k}\right) x_{k}, & a_{k}-1 \leq t \leq a_{k}, \\ x_{k}, & a_{k} \leq t \leq b_{k}, \\ \left(-t+b_{k}+1\right) x_{k}, & b_{k} \leq t \leq b_{k}+1 .\end{cases}
$$

Again $\int_{a_{k}-1}^{b_{k}+1}\left|\varphi_{k}^{\prime}(t)\right|^{2} d t=2$, and we have

$$
\begin{aligned}
\int_{a_{k}-1}^{b_{k}+1} \varphi_{k}^{*}(t) Q(t) \varphi_{k}(t) d t= & \int_{a_{k}-1}^{a_{k}} \varphi_{k}^{*}(t) Q(t) \varphi_{k}(t) d t \\
& +\int_{b_{k}}^{b_{k}+1} \varphi_{k}^{*}(t) Q(t) \varphi_{k}(t) d t+\int_{a_{k}}^{b_{k}} \varphi_{k}^{*}(t) Q(t) \varphi_{k}(t) d t \\
= & \int_{a_{k}}^{b_{k}} x_{k}^{*} Q(t) x_{k} d t+\int_{a_{k}-1}^{a_{k}}\left(t+1-a_{k}\right)^{2} x_{k}^{*} Q(t) x_{k} d t \\
& +\int_{b_{k}}^{b_{k}+1}\left(b_{k}+1-t\right)^{2} x_{k}^{*} Q(t) x_{k} d t \\
\geq & k-M\left\{\int_{a_{k}-1}^{a_{k}}\left(t+1-a_{k}\right)^{2} d t+\int_{b_{k}}^{b_{k}+1}\left(b_{k}+1-t\right)^{2}\right\} d t \\
= & k-\frac{2}{3} M
\end{aligned}
$$


and so

$$
\int_{b_{k}}^{b_{k}+1}\left\{\left|\varphi_{k}^{\prime}(t)\right|^{2}-\varphi_{k}^{*}(t) Q(t) \varphi_{k}(t)\right\} d t \leq 2+\frac{2}{3} M-k<0
$$

for $k$ sufficiently large, and again we conclude that (1.1) is oscillatory.

PROOF OF THEOREM 3.2. Applying the techniques of the previous theorem to the scalar equation

$$
y^{\prime \prime}(t)+q_{i j}(t) y(t)=0
$$

there exist intervals $\left[a_{m}, b_{m}\right]$ with $\lim _{m \rightarrow \infty} a_{m}=\infty$ and a scalar function $\varphi_{m} \in$ $A_{1}\left(a_{m}, b_{m}\right)$ (with $n=1$ ) such that

$$
\int_{a_{m}}^{b_{m}}\left\{\left|\varphi_{m}^{\prime}(t)\right|^{2}-q_{i j}(t)\left|\varphi_{m}(t)\right|^{2}\right\} d t<0 .
$$

Define $\psi_{m} \in A_{1}\left(a_{m}, b_{m}\right)$ by

$$
\psi_{m}(t)=\operatorname{col}\left(0, \ldots, \varphi_{m}(t), 0, \ldots, \varphi_{m}(t), \ldots, 0\right)
$$

where the only nonzero entries are the $i$ th and $j$ th. Then

$$
\int_{a_{m}}^{b_{m}}\left\{\left|\psi_{m}^{\prime}(t)\right|^{2}-\psi_{m}^{*}(t) Q(t) \psi_{m}(t)\right\} d t=2 \int_{a_{m}}^{b_{m}}\left\{\left|\varphi_{m}^{\prime}(t)\right|^{2}-q_{i j}(t)\left|\varphi_{m}(t)\right|^{2}\right\} d t<0
$$

implying oscillation for (1.1). A similar argument was used in [9].

PROOF OF THEOREM 3.3 . Let $m \geq a+1$ be a positive integer. Without loss of generality, we may assume that $0<\varepsilon_{m}<1$. Let

$$
\max _{m-\varepsilon_{m} / 2 \leq t \leq m}\|Q(t)\|=\alpha_{m}
$$

and choose the positive integer $k=k_{m}$ so that

$$
k>2+\frac{1}{12} \alpha_{m} \varepsilon_{m}^{2} \text {. }
$$

Let $x_{m k}$ be the unit vector given by the hypothesis of the theorem. Since $\mu\left(S_{m k}\right) \geq$ $\varepsilon_{m}$, we may find a finite collection of closed intervals $I_{j}=\left[a_{j}, b_{j}\right], 1 \leq j \leq r$, where $m<a_{1}<b_{1}<\cdots<a_{r}<b_{r}$, such that

$$
\bigcup_{j=1}^{r} I_{j} \subset S_{m k} \quad \text { and } \quad \mu\left(\bigcup_{j=1}^{r} I_{j}\right)=\sum_{j=1}^{r} \delta_{j} \geq \frac{1}{2} \varepsilon_{m}
$$

where $\delta_{j}=b_{j}-a_{j}$. Since $\int_{m}^{t} Q(s) d s$ is a continuous function of $t$, we may, by maximizing the lengths of the intervals, assume that

$$
\begin{gathered}
x_{m k}^{*}\left(\int_{m}^{a_{j}} Q(s) d s\right) x_{m k}=k, \quad 1 \leq j \leq r, \\
x_{m k}^{*}\left(\int_{m}^{b_{j}} Q(s) d s\right) x_{m k}=k, \quad 1 \leq j \leq r-1 .
\end{gathered}
$$

Furthermore, by discarding intervals if necessary, and shrinking the final interval, we may suppose that

$$
\sum_{j=1}^{r} \delta_{j}=\frac{1}{2} \varepsilon_{m}
$$


Let $a_{0}$ and $b_{0}$ be defined to be $m, \delta_{0}$ to be 0 , and let $\Delta_{j}=\sum_{i=0}^{j} \delta_{i}, j=0,1, \ldots, r$.

We define $\varphi_{m} \in A_{1}\left(m-\frac{1}{2} \varepsilon_{m}, b_{r}\right)$ as follows:

$$
\varphi_{m}(t)=\rho_{m}(t) x_{m k},
$$

where

$$
\rho_{m}= \begin{cases}t+\frac{1}{2} \varepsilon_{m}-m, & m-\frac{1}{2} \varepsilon_{m} \leq t \leq m \\ \frac{1}{2} \varepsilon_{m}-\Delta_{j}, & b_{j} \leq t \leq a_{j+1} ; \quad 0 \leq j \leq r-1 \\ \frac{1}{2} \varepsilon_{m}-\Delta_{j-1}+a_{j}-t, & a_{j} \leq t \leq b_{j+1} ; \quad 0 \leq j \leq r .\end{cases}
$$

We have

$$
\int_{m-\varepsilon_{m} / 2}^{b_{r}}\left|\varphi_{m}^{\prime}(t)\right|^{2} d t=\frac{1}{2} \varepsilon_{m}+\Delta_{r}=\varepsilon_{m}
$$

and

$$
\begin{aligned}
& \int_{m-\varepsilon_{m} / 2}^{b_{r}} \varphi_{m}^{*}(t) Q(t) \varphi_{m}(t) d t \\
& =\left(\int_{m-\varepsilon_{m} / 2}^{b_{r}}+\sum_{j=0}^{r-1} \int_{b_{j}}^{a_{j+1}}+\sum_{j=1}^{r-1} \int_{a_{j}}^{b_{j}}\right) \varphi_{m}^{*}(t) Q(t) \varphi_{m}(t) d t .
\end{aligned}
$$

By (6.10) and (6.11),

$$
\begin{aligned}
\int_{m-\varepsilon_{m} / 2}^{m} \varphi_{m}^{*}(t) Q(t) \varphi_{m}(t) d t & \geq-\alpha_{m} \int_{m-\varepsilon_{m} / 2}^{m}\left(t+\frac{1}{2} \varepsilon_{m}-m\right)^{2} d t \\
& =-\frac{1}{24} \alpha_{m} \varepsilon_{m}^{3} \\
\int_{b_{0}}^{a_{1}} \varphi_{m}^{*}(t) Q(t) \varphi_{m}(t) d t & =\frac{1}{2} \varepsilon_{m} \int_{m}^{a_{1}} x_{m k}^{*} Q(t) x_{m k} d t \\
\geq & \frac{1}{2} \varepsilon_{m} x_{m k}^{*}\left(\int_{m}^{a_{1}} Q(t) d t\right) x_{m k}=\frac{1}{2} \varepsilon_{m} k
\end{aligned}
$$

by $(6.10)$.

For $1 \leq j \leq r-1$,

$$
\begin{aligned}
& \int_{b_{j}}^{a_{j+1}} \varphi_{m}^{*}(t) Q(t) \varphi_{m}(t) d t=\left(\frac{1}{2} \varepsilon_{m}-\Delta_{j}\right) \int_{b_{j}}^{a_{j+1}} x_{m k}^{*} Q(t) x_{m k} d t \\
& =\left(\frac{1}{2} \varepsilon_{m}-\Delta_{j}\right) \int_{m}^{a_{j}} x_{m k}^{*} Q(t) x_{m k} d t-\int_{m}^{b_{j}} x_{m k}^{*} Q(t) x_{m k} d t=0
\end{aligned}
$$

by (6.10) and (6.11).

Noting that for $1 \leq j \leq r, \rho_{m}(t) \geq 0$, and $\rho_{m}^{\prime}(t) \equiv-1$ on $\left[a_{j}, b_{j}\right]$, we have, on integrating by parts,

$$
\begin{aligned}
& \int_{a_{j}}^{b_{j}} \varphi_{m}^{*}(t) Q(t) \varphi_{m}(t) d t=\int_{a_{j}}^{b_{j}} \rho_{m}^{2}(t) x_{m k}^{*} Q(t) x_{m k} d t \\
& \quad=\rho_{m}^{2}\left(b_{j}\right) \int_{a_{j}}^{b_{j}} x_{m k}^{*} Q(t) x_{m k} d t+\int_{a_{j}}^{b_{j}} 2 \rho_{m}(t)\left(\int_{a_{j}}^{t} x_{m k}^{*} Q(s) x_{m k} d s\right) d t
\end{aligned}
$$


By (6.10) and (6.11),

$$
\begin{aligned}
\int_{a_{j}}^{b_{j}} x_{m k}^{*} Q(t) x_{m k} d t & =\left(\int_{m}^{b_{j}}-\int_{m}^{a_{j}}\right) x_{m k}^{*} Q(t) x_{m k} d t \\
& \geq 0 \quad \text { (with equality if } 1 \leq j \leq r-1) .
\end{aligned}
$$

Since $t \in\left[a_{j}, b_{j}\right] \Rightarrow t \in S_{m k}$, using (6.10) we find that for $t \in\left[a_{j}, b_{j}\right]$,

$$
\int_{a_{j}}^{t} x_{m k}^{*} Q(s) x_{m k} d s=\left(\int_{m}^{t}-\int_{m}^{a_{j}}\right) x_{m k}^{*} Q(s) x_{m k} d s \geq 0 .
$$

From (6.20)-(6.22), we have

$$
\int_{a_{j}}^{b_{j}} \varphi_{m}^{*}(t) Q(t) \varphi_{m}(t) d t \geq 0, \quad j=1, \ldots, r .
$$

From (6.15)-(6.19) and (6.23), we see that

$$
\int_{m-\varepsilon_{m} / 2}^{b_{r}}\left\{\left|\varphi_{m}^{\prime}(t)\right|^{2}-\varphi_{m}^{*}(t) Q(t) \varphi_{m}(t)\right\} d t \leq \varepsilon_{m}+\frac{1}{24} \alpha_{m} \varepsilon_{m}^{3}-\frac{1}{2} \varepsilon_{m} k<0
$$

by (6.9). Since $m-\frac{1}{2} \varepsilon_{m}>m-\frac{1}{2}$ and $m$ is an arbitrary positive integer exceeding $a$, the theorem follows.

PROOF OF THEOREM 3.4. Since (3.6) is oscillatory, there exist subintervals $\left[\tilde{a}_{m}, \tilde{b}_{m}\right]$ of $[a, A)$ with $\lim _{m \rightarrow \infty} \tilde{a}_{m}=A$, and $\tilde{\varphi}_{m} \in\left(\tilde{a}_{m}, \tilde{b}_{m}\right)$ such that

$$
\int_{\tilde{a}_{m}}^{\tilde{b}_{m}}\left\{\left|\tilde{\varphi}_{m}^{\prime}(t)\right|^{2}-\tilde{\varphi}_{m}^{*}(t) \tilde{Q}(t) \tilde{\varphi}_{m}(t)\right\} d t<0, \quad m=1,2, \ldots
$$

Choose $a_{m}, b_{m} \geq a$ so that if $Q_{m}$ is the restriction of $Q$ to $\left[a_{m}, b_{m}\right)$ and $\tilde{Q}_{m}$ is the restriction of $\tilde{Q}$ to $\left[\tilde{a}_{m}, \tilde{b}_{m}\right)$, and if $S_{m}=S \cap\left[a_{m}, b_{m}\right)$, then $\tilde{Q}_{m}=T_{S_{m}}\left(Q_{m}\right)$. Then $\lim _{m \rightarrow \infty} a_{m}=\infty$. Let $\varphi_{m}$ by defined on $\left[a_{m}, b_{m}\right)$ so that $\varphi_{m}$ is continuous and $\tilde{\varphi}_{m}=T_{S_{m}}\left(\varphi_{m}\right)$. Extend $\varphi_{m}$ to $\left[a_{m}, b_{m}\right]$ by defining $\varphi_{m}\left(b_{m}\right)=0$. Then $\varphi_{m} \in A_{1}\left(a_{m}, b_{m}\right)$ and $\varphi_{m}$ is constant on each component interval of $\left[a_{m}, b_{m}\right) \backslash S_{m}$. We have

$$
\begin{aligned}
& \int_{a_{m}}^{b_{m}}\left\{\left|\varphi_{m}^{\prime}(t)\right|^{2}-\varphi_{m}^{*}(t) Q(t) \varphi_{m}(t)\right\} d t \\
& \quad=\int_{\tilde{a}_{m}}^{\tilde{b}_{m}}\left\{\left|\tilde{\varphi}_{m}^{\prime}(t)\right|^{2}-\tilde{\varphi}_{m}^{*}(t) \tilde{Q}(t) \tilde{\varphi}_{m}(t)\right\} d t-\sum \int_{\alpha_{i}}^{\beta_{i}} \varphi_{m}^{*}(t) Q(t) \varphi_{m}(t) d t
\end{aligned}
$$

where the summation is over indices $i$ for which $\left(\alpha_{i}, \beta_{i}\right) \subset\left[a_{m}, b_{m}\right)$. By (6.25) and the nonnegative definiteness condition (3.5), the right-hand side of (6.26) is negative. Thus (1.1) is oscillatory.

\section{REFERENCES}

1. K. Akiyama, On the maximum eigenvalue conjecture for the oscillation of second order differential systems, M. Sc. Thesis, Univ. of Ottawa, Canada, 1983.

2. W. Allegretto and L. Erbe, Oscillation criteria for matrix differential inequalities, Canad. Math. Bull. 16 (1973), 5-10. 
3. F. V. Atkinson, H. G. Kaper and M. K. Kwong, An oscillation criterion for linear second-order differential systems, Proc. Amer. Math. Soc. 94 (1985), 91-96.

4. G. J. Butler and L. H. Erbe, Oscillation results for second order differential systems, SIAM J. Math. Anal. 17 (1986), 19-29.

5. __ Oscillation results for self-adjoint differential systems, J. Math. Anal. Appl. 115 (1986), 470-481.

6. R. Byers, B. J. Harris and M. K. Kwong, Weighted means and oscillation conditions for second order matrix differential equations, J. Differential Equations 61 (1986), 164-177.

7. W. A. Coppel, Stability and asymptotic behavior of differential equations, Heath, Boston, Mass., 1965.

8. G. J. Etgen and R. T. Lewis, Positive functionals and oscillation criteria for second order differential systems, Proc. Edinburgh Math. Soc. 22 (1979), 277-290.

9. W. B. Fite, Concerning the zeros of the solutions of certain differential equations, Trans. Amer. Math. Soc. 19 (1918), 341-352.

10. S. Halvorsen and A. B. Mingarelli, preprint, 1984.

11. P. Hartman, Oscillation criteria for self-adjoint second order differential systems and "Principal sectional curvatures", J. Differential Equations 34 (1979), 326-338.

12. __ Ordinary differential equations, Wiley, New York, 1964.

13. D. B. Hinton and R. T. Lewis, Oscillation theory of generalized second order differential equations, Rocky Mountain J. Math 10 (1980), 751-766.

14. M. K. Kwong and H. G. Kaper, Oscillation of two-dimensional linear second order differential systems, J. Differential Equations 56 (1985), 195-205.

15. M. K. Kwong and A. Zettl, A new approach to second order linear oscillation theory, Proc. Conf. Ordinary Differential Equations and operators (Dundee, 1982), Lecture Notes in Math., vol. 1032, Springer, Berlin and New York, 1983, pp. 328-345.

16. __ Integral inequalities and second order linear oscillation, J. Differential Equations 45 (1982), 16-33.

17. M. K. Kwong, H. G. Kaper, K. Akiyama and A. B. Mingarelli, Oscillation of second order differential systems, Proc. Amer. Math. Soc. 91 (1984), 85-91.

18. A. B. Mingarelli, On a conjecture for oscillation of second order differential systems, Proc. Amer. Math. Soc. 82 (1981), 593-598.

19. _ An oscillation criterion for second order self-adjoint differential systems, C. R. Math. Rep. Acad. Sci. Canada 2 (1980), 287-290.

20. R. A. Moore, The behavior of solutions of a linear differential equation of second order, Pacific J. Math. 5 (1955), 125-145.

21. C. Olech, Z. Opial and T. Ważewski, Sur le problème d'oscillation des intégrales de l'équation $y^{\prime \prime}+q(t) y=0$, Bull. Acad. Polon. Sci. Cl. III 5 (1957), 621-626.

22. B. N. Parlett, The symmetric eigenvalue problem, Prentice-Hall, Englewood Cliffs, N.J., 1980.

23. M. Rab, Kriterien fur die Oszillation der Lösungen der Differentialgleichung $\left(p(x) y^{\prime}\right)^{\prime}+q(x) y=$ 0, Casobis. Pest. Mat. 84 (1959), 335-370.

24. C. A. Swanson, Comparison and oscillation theory of linear differential equations, Academic Press, New York, 1968.

25. E. C. Tomastik, Oscillation of systems of second order differential equations, J. Differential Equations 9 (1971), 436-442.

26. T. Walters, A characterization of positive linear functionals and oscillation criteria for matrix differential equations, Proc. Amer. Math. Soc. 78 (1980), 198-202.

27. D. Willett, Classification of second order linear differential equations with respect to oscillation, Adv. in Math. 3 (1969), 594-623.

28. _ On the oscillatory behavior of the solutions of second order linear differential equations, Ann. Polon. Math. 21 (1969), 175-194.

29. A. Wintner, A criterion of oscillatory stability, Quart. Appl. Math. 7 (1949), 115-117.

30. J. S. W. Wong, Oscillation and nonoscillation of solutions of second order linear differential equations with integrable coefficients, Trans. Amer. Math. Soc. 144 (1969), 197-215.

Department of Mathematics, University of Alberta, Edmonton, Alberta, CANADA

Department of Mathematics, University of Ottawa, Ottawa, Ontario, CANADA 